\title{
Reaction of Triplet Phenylnitrene with Molecular Oxygen
}

\author{
Joel Mieres-Pérez, Enrique Mendez-Vega, Kavitha Velappan, and Wolfram Sander* \\ Lehrstuhl für Organische Chemie II, Ruhr-Universität Bochum, D-44780 Bochum, Germany
}

Content:

Absolute energies and Cartesian coordinates of optimized structures

$2-4$

Figure S1 Photochemistry of phenylazide 3 in Ar

Figure S2 IR spectrum of FVT $\left(300^{\circ} \mathrm{C}\right)$ products of 3 in $\mathrm{Ar}$

Figure S3 IR spectrum of FVT $\left(420^{\circ} \mathrm{C}\right)$ products of $\mathbf{3}$ in $\mathrm{Ar}$

Figure S4 Minor products of FVT of 3

Figure S5 IR spectrum showing the presence of cyanocyclopentadiene $\mathbf{1 0}$

Figure S6 IR spectra showing the conversion of nitrene 1a to nitrobenzene 8a in both ${ }^{16} \mathrm{O}_{2}$ and ${ }^{18} \mathrm{O}_{2}$ experiments

Figure S7 Comparison between experimental spectrum for the thermal reaction and calculated spectrum for dioxaziridine $\mathbf{7 a}$

Figure S8 IR spectra showing the reaction of $1 \mathrm{a}$ in $4 \% \mathrm{O}_{2}$-doped $\mathrm{Xe}$ at $30 \mathrm{~K}$

Figure S9 IR spectra showing the reaction of $1 \mathrm{a}$ in $4 \% \mathrm{O}_{2}$-doped Ar at 30-35 K

Figure S10 IR spectra showing the reaction of $1 \mathbf{a}$ in $4 \%{ }^{16} \mathrm{O}_{2}$ and

${ }^{18} \mathrm{O}_{2}$-doped $\mathrm{Xe}$ at $30 \mathrm{~K}$

Tables (S1-S7) Calculated frequencies 
Absolute energies and Cartesian coordinates of optimized structures

\section{phenylnitrene 1a}

\begin{tabular}{|c|c|c|c|c|}
\hline$\dot{N} \bullet$ & \multicolumn{4}{|c|}{$\begin{array}{l}\text { Method: B3LYP/aug-cc-pVTZ } \\
\text { Charge: } 0 \\
\text { Multiplicity: } 3 \\
\text { Symmetry: } \mathrm{C}_{2 \mathrm{v}} \\
\mathrm{E}(\mathrm{UB}+\mathrm{HF}-\mathrm{LYP})=-286.410224 \text { a.u. } \\
\mathrm{E}(\mathrm{UB}+\mathrm{HF}-\mathrm{LYP})+\mathrm{ZPE}=-286.319175 \text { a.u. }\end{array}$} \\
\hline & $\mathrm{C}$ & 0.00000000 & 1.21200200 & -1.04140392 \\
\hline & $\mathrm{C}$ & 0.00000000 & 0.00000000 & -1.73941192 \\
\hline & $\mathrm{C}$ & 0.00000000 & -1.21200200 & -1.04140392 \\
\hline & $\mathrm{C}$ & 0.00000000 & -1.22908300 & 0.33556808 \\
\hline & $\mathrm{C}$ & 0.00000000 & 0.00000000 & 1.06865108 \\
\hline & $\mathrm{C}$ & 0.00000000 & 1.22908300 & 0.33556808 \\
\hline & $\mathrm{H}$ & 0.00000000 & 2.14567700 & -1.58762292 \\
\hline & $\mathrm{H}$ & 0.00000000 & 0.00000000 & -2.82041092 \\
\hline & $\mathrm{H}$ & 0.00000000 & -2.14567700 & -1.58762292 \\
\hline & $\mathrm{H}$ & 0.00000000 & -2.15773600 & 0.88775908 \\
\hline & $\mathrm{H}$ & 0.00000000 & 2.15773600 & 0.88775908 \\
\hline & $\mathrm{N}$ & 0.00000000 & 0.00000000 & 2.38781908 \\
\hline
\end{tabular}

\section{phenylazide 3}

\begin{tabular}{|c|c|c|c|c|}
\hline $\mathrm{N}_{3}$ & \multicolumn{4}{|c|}{$\begin{array}{l}\text { Method: B3LYP/aug-cc-pVTZ } \\
\text { Charge: } 0 \\
\text { Multiplicity: } 1 \\
\text { Symmetry: } C_{s} \\
\text { E(RB+HF-LYP })=-395.984783 \text { a.u. } \\
\text { E(RB+HF-LYP) + ZPE }=-395.881431 \text { a.u. }\end{array}$} \\
\hline & $\mathrm{C}$ & -0.14854191 & -0.36228202 & 0.00000000 \\
\hline & $\mathrm{C}$ & 0.87922210 & -1.30476400 & 0.00000000 \\
\hline & $\mathrm{C}$ & 2.20030110 & -0.88276098 & 0.00000000 \\
\hline & $\mathrm{C}$ & 1.47959406 & 1.40960801 & 0.00000000 \\
\hline & $\mathrm{C}$ & 0.15234406 & 1.00049899 & 0.00000000 \\
\hline & $\mathrm{N}$ & -1.47007290 & -0.87713404 & 0.00000000 \\
\hline & $\mathrm{N}$ & -2.41096192 & -0.08759306 & 0.00000000 \\
\hline & $\mathrm{N}$ & -3.35519893 & 0.53078392 & 0.00000000 \\
\hline & $\mathrm{H}$ & 0.62722312 & -2.35564000 & 0.00000000 \\
\hline & $\mathrm{H}$ & -0.63982795 & 1.73739597 & 0.00000000 \\
\hline & $\mathrm{C}$ & 2.50811707 & 0.47433203 & 0.00000000 \\
\hline & $\mathrm{H}$ & 3.53899707 & 0.79951705 & 0.00000000 \\
\hline & $\mathrm{H}$ & 2.99317211 & -1.61829796 & 0.00000000 \\
\hline & $\mathrm{H}$ & 1.70785304 & 2.46683501 & 0.00000000 \\
\hline
\end{tabular}


syn-nitroso oxide (syn-6a)

\begin{tabular}{|l|ll|}
\hline & \multicolumn{3}{l}{ Method: B3LYP/aug-cc-pVTZ } \\
Charge: 0 \\
Multiplicity: 1
\end{tabular}

anti-nitroso oxide (anti-6a)

\begin{tabular}{|l|lll|}
\hline & \multicolumn{3}{l|}{ Method: B3LYP/aug-cc-pVTZ } \\
Charge: 0 & \\
Multiplicity: 1 & \\
Symmetry: $\mathrm{C}_{\mathrm{s}}$ & & \\
$\mathrm{E}(\mathrm{RB}+\mathrm{HF}-\mathrm{LYP})=-436.810584$ a.u. \\
$\mathrm{E}(\mathrm{RB}+\mathrm{HF}-\mathrm{LYP})+\mathrm{ZPE}=-436.709991$ & $\mathrm{a} . \mathrm{u}$. \\
\hline & & & \\
$\mathrm{C}$ & -0.07380801 & -0.24436813 & 0.00000000 \\
$\mathrm{C}$ & 0.37412087 & 1.08899591 & 0.00000000 \\
$\mathrm{~N}$ & -1.40262597 & -0.66134025 & 0.00000000 \\
$\mathrm{C}$ & 1.73190985 & 1.34388003 & 0.00000000 \\
$\mathrm{C}$ & 2.65314294 & 0.29418811 & 0.00000000 \\
$\mathrm{C}$ & 2.21056306 & -1.02392793 & 0.00000000 \\
$\mathrm{C}$ & 0.85239308 & -1.29686605 & 0.00000000 \\
$\mathrm{H}$ & -0.33564320 & 1.90213085 & 0.00000000 \\
$\mathrm{H}$ & 2.08255776 & 2.36668606 & 0.00000000 \\
$\mathrm{H}$ & 3.71281892 & 0.50914120 & 0.00000000 \\
$\mathrm{H}$ & 2.92292213 & -1.83690787 & 0.00000000 \\
$\mathrm{H}$ & 0.47985217 & -2.31121008 & 0.00000000 \\
$\mathrm{O}$ & -2.20704106 & 0.32960668 & 0.00000000 \\
$\mathrm{O}$ & -3.48471604 & 0.04890957 & 0.00000000 \\
\hline
\end{tabular}


dioxaziridine 7a

\begin{tabular}{|c|c|c|c|c|}
\hline $\begin{array}{c}\text { O-O } \\
\text { N } \\
1\end{array}$ & \multicolumn{4}{|c|}{$\begin{array}{l}\text { Method: B3LYP/aug-cc-pVTZ } \\
\text { Charge: } 0 \\
\text { Multiplicity: } 1 \\
\text { Symmetry: } \mathrm{C}_{\mathrm{s}} \\
\mathrm{E}(\mathrm{RB}+\mathrm{HF}-\mathrm{LYP})=-436.789734 \text { a.u. } \\
\mathrm{E}(\mathrm{RB}+\mathrm{HF}-\mathrm{LYP})+\mathrm{ZPE}=-436.689519 \text { a.u. }\end{array}$} \\
\hline & $\mathrm{C}$ & 1.55702030 & 1.35215119 & 0.00000000 \\
\hline & $\mathrm{C}$ & 2.50026981 & 0.32486882 & 0.00000000 \\
\hline & $\mathrm{C}$ & 2.09023788 & -1.00185559 & 0.00000000 \\
\hline & $\mathrm{C}$ & 0.73315644 & -1.30675662 & 0.00000000 \\
\hline & $\mathrm{C}$ & -0.19514606 & -0.27488927 & 0.00000000 \\
\hline & $\mathrm{C}$ & 0.20279188 & 1.05927316 & 0.00000000 \\
\hline & $\mathrm{H}$ & 1.88245380 & 2.38335771 & 0.00000000 \\
\hline & $\mathrm{H}$ & 2.82116672 & -1.79834165 & 0.00000000 \\
\hline & $\mathrm{H}$ & 0.38423495 & -2.32982711 & 0.00000000 \\
\hline & $\mathrm{H}$ & -0.54014399 & 1.84210324 & 0.00000000 \\
\hline & $\mathrm{N}$ & -1.57196069 & -0.70924927 & 0.00000000 \\
\hline & $\mathrm{O}$ & -2.40181333 & 0.21168308 & 0.72805019 \\
\hline & $\mathrm{O}$ & -2.40181333 & 0.21168308 & -0.72805019 \\
\hline & $\mathrm{H}$ & 3.55504516 & 0.56377328 & 0.00000000 \\
\hline
\end{tabular}

nitrobenzene 8a

\begin{tabular}{|c|c|c|c|c|}
\hline $\mathrm{O}_{=N}^{\oplus} \mathrm{O}^{\Theta}$ & \multicolumn{4}{|c|}{$\begin{array}{l}\text { Method: B3LYP/aug-cc-pVTZ } \\
\text { Charge: } 0 \\
\text { Multiplicity: } 1 \\
\text { Symmetry: } \mathrm{C}_{2 \mathrm{v}} \\
\mathrm{E}(\mathrm{RB}+\mathrm{HF}-\mathrm{LYP})=-436.917926 \text { a.u. } \\
\mathrm{E}(\mathrm{RB}+\mathrm{HF}-\mathrm{LYP})+\mathrm{ZPE}=-436.815050 \text { a.u. }\end{array}$} \\
\hline & $\mathrm{C}$ & 0.00000000 & 0.00000000 & -2.50959884 \\
\hline & $\mathrm{C}$ & 0.00000000 & 1.20696600 & -1.81706484 \\
\hline & $\mathrm{C}$ & 0.00000000 & 1.21503000 & -0.42926884 \\
\hline & $\mathrm{C}$ & 0.00000000 & 0.00000000 & 0.24136316 \\
\hline & $\mathrm{C}$ & 0.00000000 & -1.21503000 & -0.42926884 \\
\hline & $\mathrm{C}$ & 0.00000000 & -1.20696600 & -1.81706484 \\
\hline & $\mathrm{H}$ & 0.00000000 & 0.00000000 & -3.59102184 \\
\hline & $\mathrm{H}$ & 0.00000000 & 2.14328100 & -2.35713384 \\
\hline & $\mathrm{H}$ & 0.00000000 & 2.13487000 & 0.13383616 \\
\hline & $\mathrm{H}$ & 0.00000000 & -2.13487000 & 0.13383616 \\
\hline & $\mathrm{H}$ & 0.00000000 & -2.14328100 & -2.35713384 \\
\hline & $\mathrm{N}$ & 0.00000000 & 0.00000000 & 1.71818416 \\
\hline & $\mathrm{O}$ & 0.00000000 & -1.08239200 & 2.28598416 \\
\hline & $\mathrm{O}$ & 0.00000000 & 1.08239200 & 2.28598416 \\
\hline
\end{tabular}



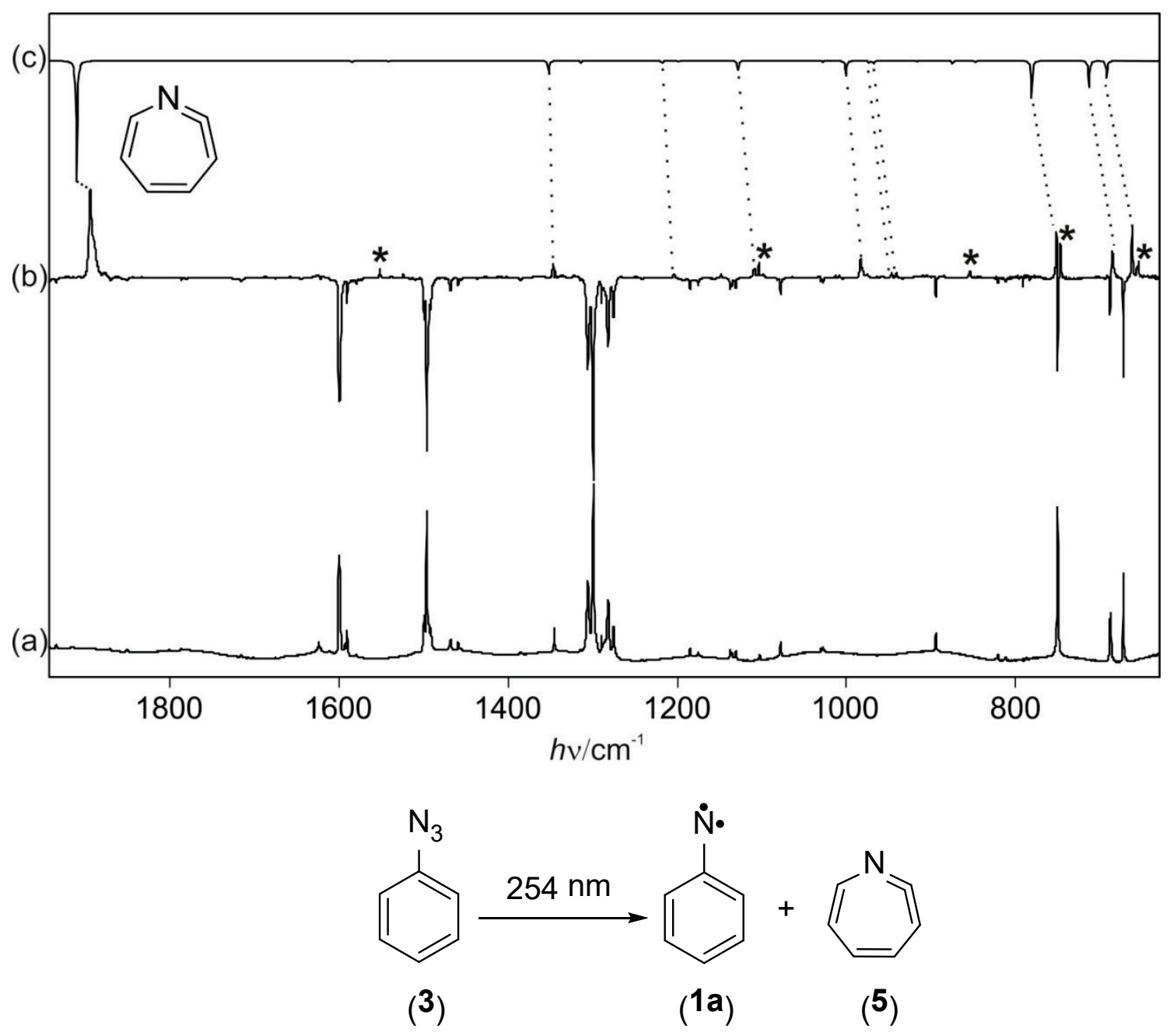

Figure S1. Photochemistry of phenylazide 3 in argon at 4 K. (a) Deposition spectrum of phenylazide 3 in Ar matrix (600-1950 $\mathrm{cm}^{-1}$, rest of the spectral region is removed for clarity). (b) Difference spectrum after irradiation with $254 \mathrm{~nm}$ light from a low-pressure mercury arc-lamp for 30 min. Bands pointing downward belongs to the precursor and bands pointing upward belongs to the photoproducts of phenylazide $\mathbf{3}$. Bands marked with asterisks are assigned to 1a. The other peaks correspond to ketenimine 5. (c) Calculated spectrum of 5 at the B3LYP/aug-cc-pVTZ level of theory, unscaled. 

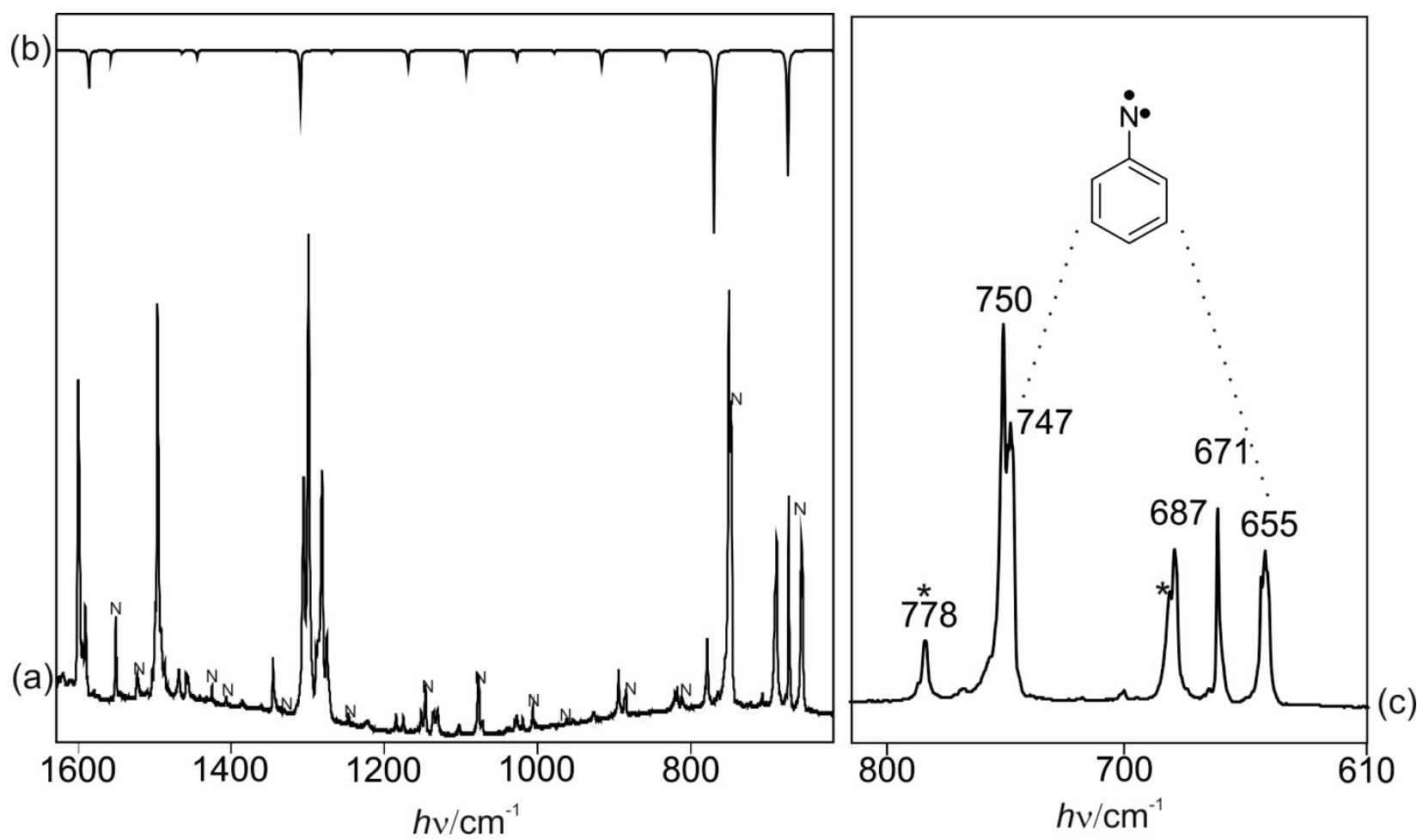

Figure S2. (a) IR spectrum of thermolysis products $\left(300^{\circ} \mathrm{C}\right)$ of phenylazide 3 in Ar matrix at $4 \mathrm{~K}$. (b) Calculated spectrum of $1 \mathbf{a}$ at the B3LYP/aug-cc-pVTZ level of theory, unscaled. The spectrum is in good agreement with the calculated values for 1a. (c) $610-800 \mathrm{~cm}^{-1}$ region of the same spectrum. The two most intense bands of $1 \mathbf{a}$ at 747 and $655 \mathrm{~cm}^{-1}$ are present in the spectrum, 1a bands are labelled as $\mathrm{N}$. The bands at 690 and $778 \mathrm{~cm}^{-1}$ correspond to azobenzene 9. Bands at 750,687 and $671 \mathrm{~cm}^{-1}$ correspond to non-pyrolyzed precursor. 

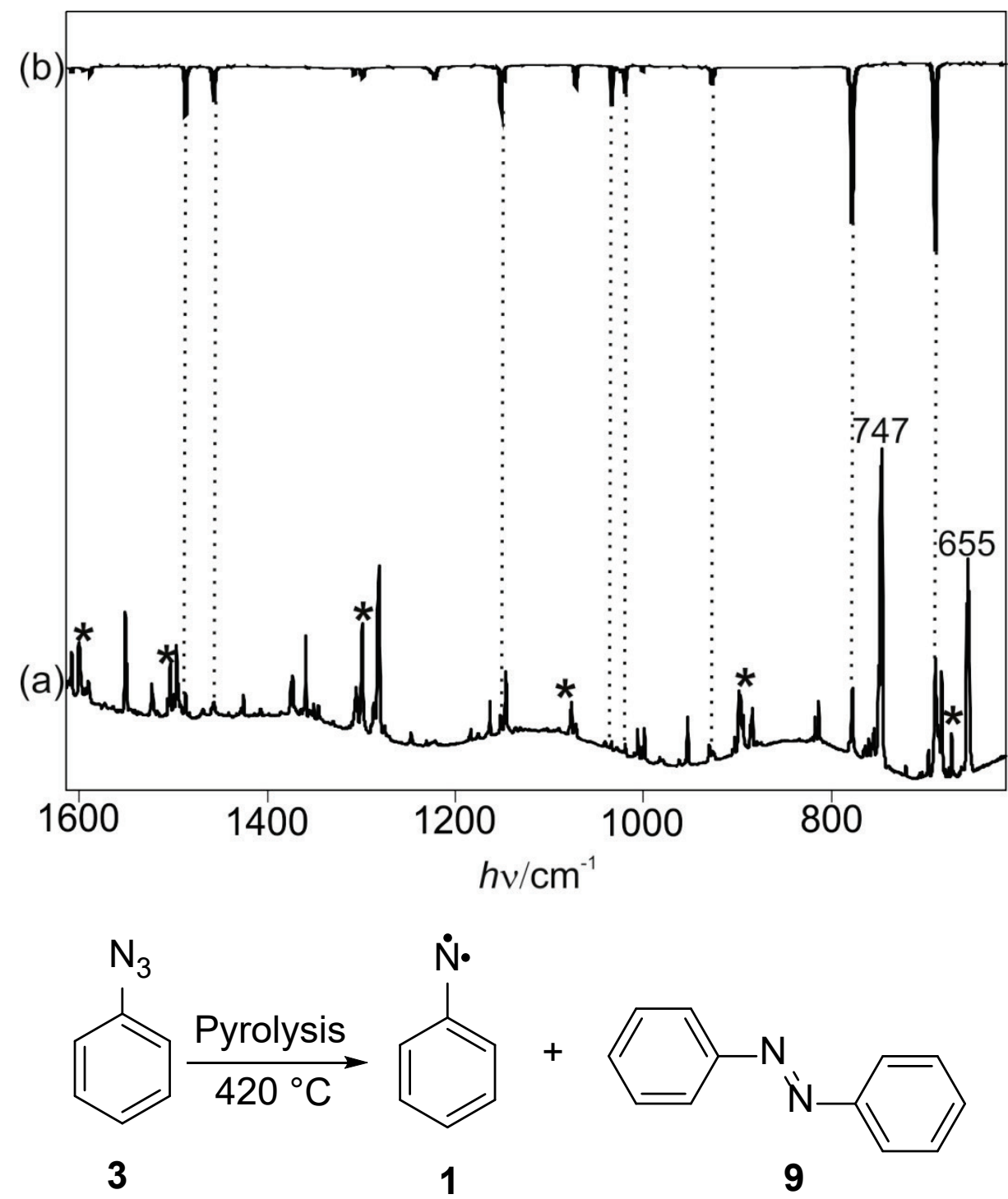

Figure S3. FVT products of 3 at $420{ }^{\circ} \mathrm{C}$ (a) IR spectrum of FVT products $\left(420{ }^{\circ} \mathrm{C}\right)$ of phenylazide 3 in Ar matrix at $4 \mathrm{~K}$. The most intense bands of $1 \mathrm{a}$ are labeled $\left(747\right.$ and $655 \mathrm{~cm}^{-1}$ ). The bands of the precursor remaining after the pyrolysis are marked with asterisks. (b) Matrix isolated spectrum of azobenzene 9 in Ar matrix at $4 \mathrm{~K}$. 


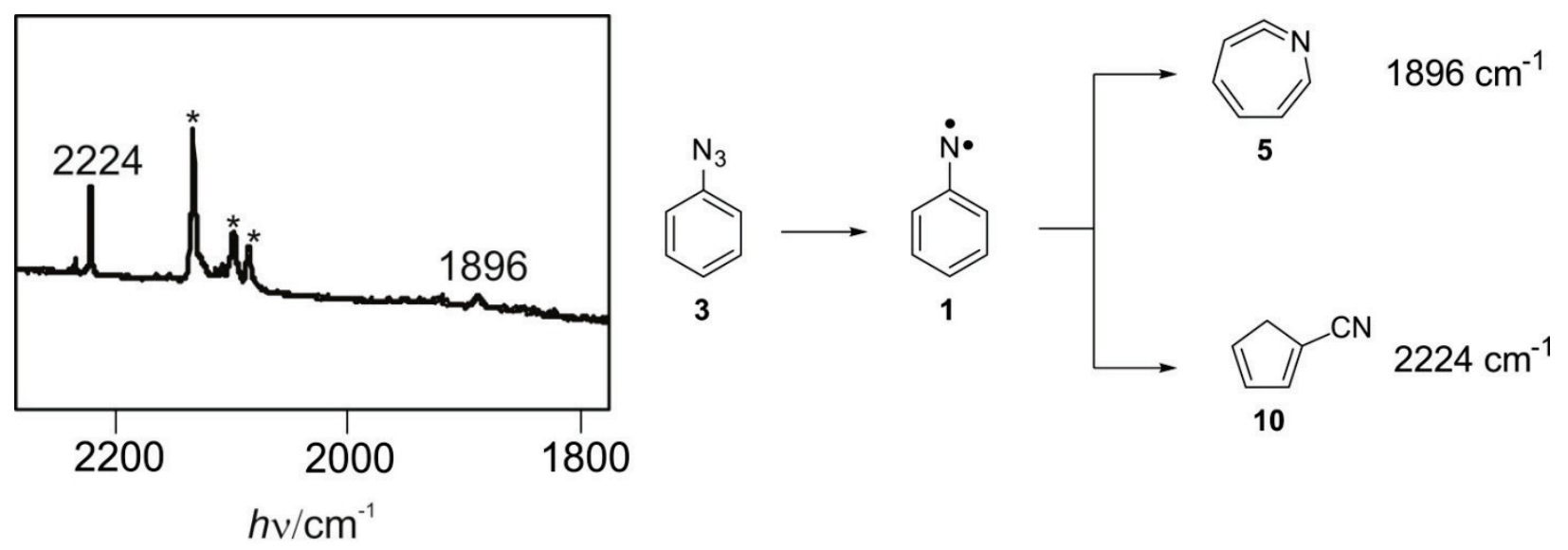

Figure S4. IR spectrum showing the characteristic bands of cyanocyclopentadiene $\mathbf{1 0}$ and ketenimine 5 which are formed as minor products between $400-500^{\circ} \mathrm{C}$ The precursor bands are marked with asterisks.

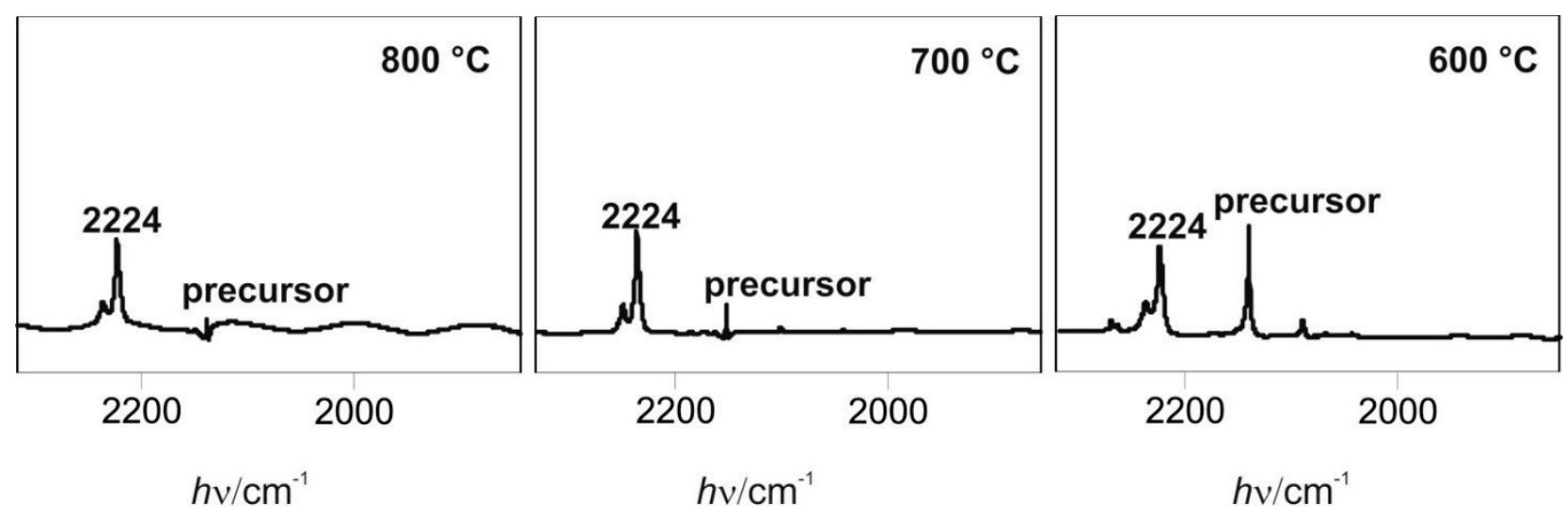

Figure S5. IR spectrum showing the presence of cyanocyclopentadiene 10. This is the main product between $600-800^{\circ} \mathrm{C}$. The remaining non-pyrolyzed precursor decreased as increasing the temperature. 


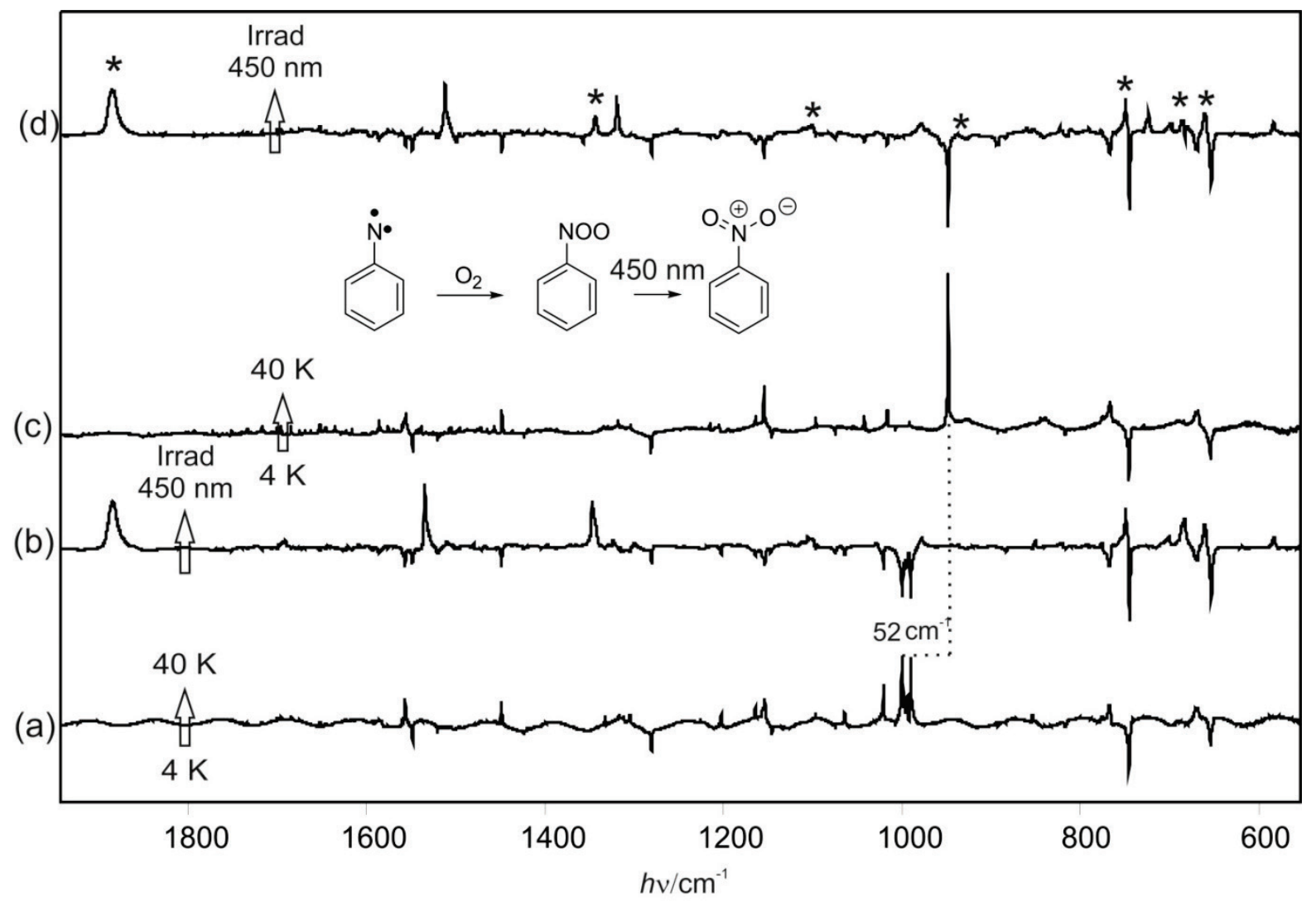

Figure S6. IR spectra showing the conversion of nitrene 1a to nitrobenzene 8a in both ${ }^{16} \mathrm{O}_{2}$ and ${ }^{18} \mathrm{O}_{2}$ experiments. (a) - (b) ${ }^{16} \mathrm{O}_{2}$. (c) - (d) ${ }^{18} \mathrm{O}_{2}$. Bands of 5 marked with asterisks. 


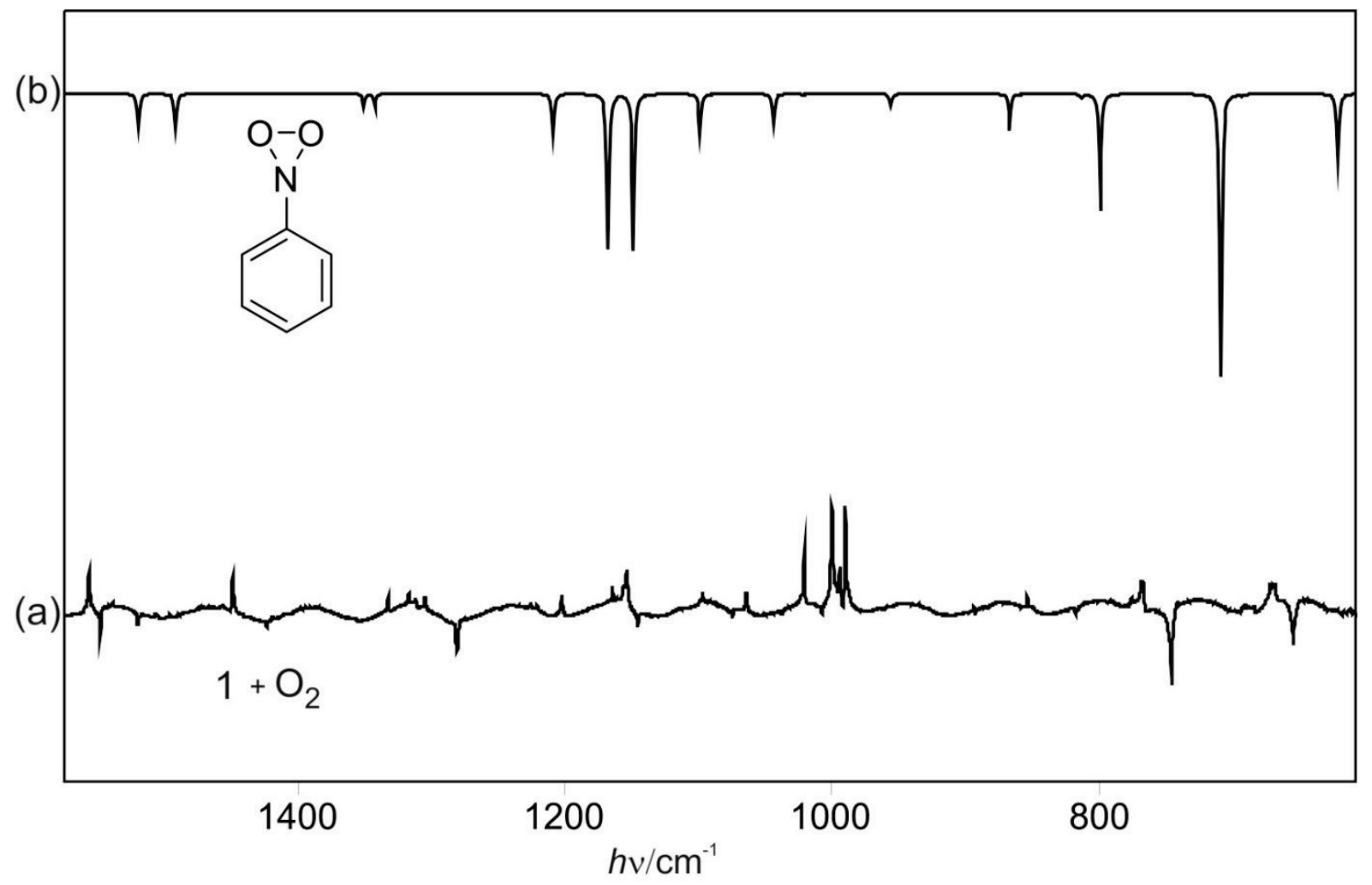

Figure S7. Comparison between experimental spectrum for the thermal reaction and calculated spectrum for dioxaziridine 7a. (a) Difference spectrum after annealing at $40 \mathrm{~K}$. (b) Calculated spectrum of dioxaziridine 7a at the B3LYP/aug-cc-pVTZ level of theory, unscaled. Dioxaziridine $7 \mathbf{a}$ is not present as thermal product of the reaction. 


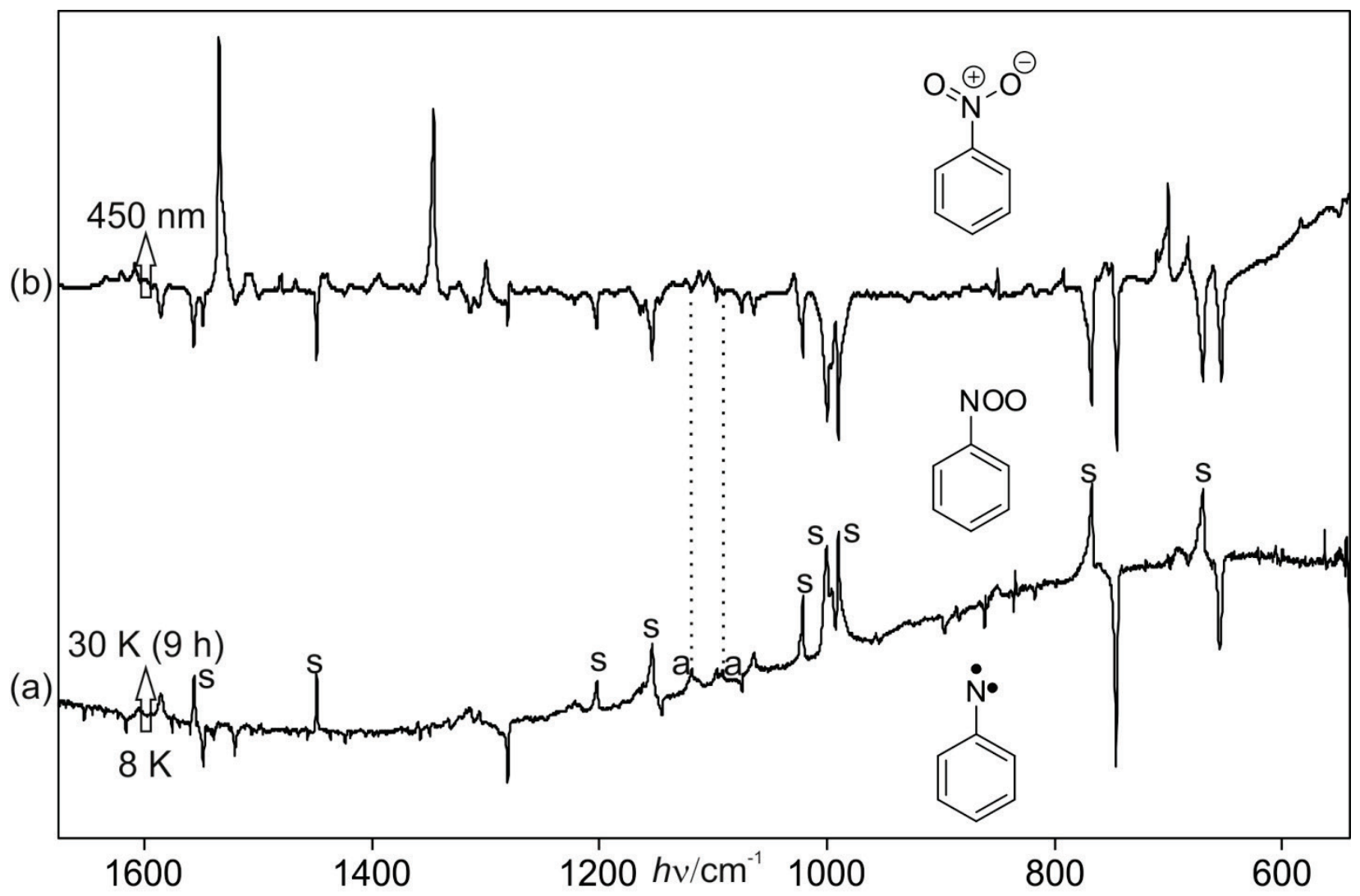

Figure S8. IR spectra showing the reaction of $\mathbf{1 a}$ in $4 \% \mathrm{O}_{2}$-doped $\mathrm{Xe}$ at $30 \mathrm{~K}$. (a) Difference spectrum after annealing at $30 \mathrm{~K}$, bands pointing downward assigned to nitrene 1a are decreasing in intensity and bands pointing upward assigned to the nitroso oxide 6a are appearing. (b) Difference spectrum after irradiation with $450 \mathrm{~nm}$, bands of $6 \mathbf{a}$ decrease in intensity and bands of nitrobenzene 8a appear concomitantly. Bands of 1a decrease also by irradiation. Bands of both isomers are labelled as $\mathbf{a}($ anti) and $\mathbf{s}(s y n)$. 


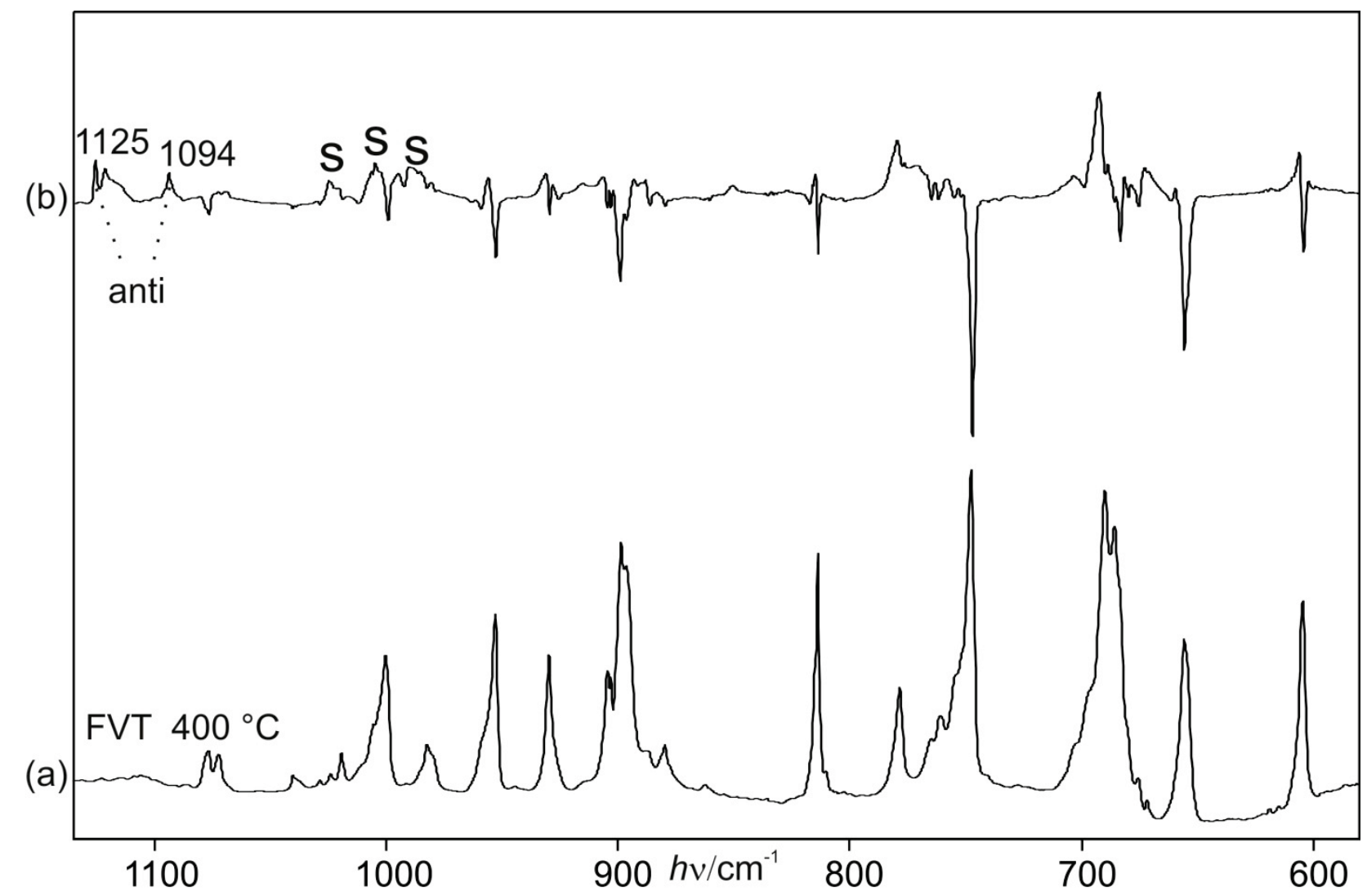

Figure S9. IR spectra showing the reaction of $1 \mathrm{a}$ in $4 \% \mathrm{O}_{2}$-doped argon matrix. (a) Spectrum obtained after FVT of phenylazide 3 at $400^{\circ} \mathrm{C}$ and subsequent trapping of the products in $4 \% \mathrm{O}_{2}$-doped Ar. (b) Difference spectrum after annealing at $30-35 \mathrm{~K}$ for $2 \mathrm{~h}$ (The spectrum after annealing at $30 \mathrm{~K}$ has the same features just less intense). Bands labelled as $\mathbf{S}$ belong to the $s y n$-isomer. The predicted bands for the antiisomer are 1135 and $1103 \mathrm{~cm}^{-1}$ at the B3LYP/aug-cc-pVTZ level of theory. 


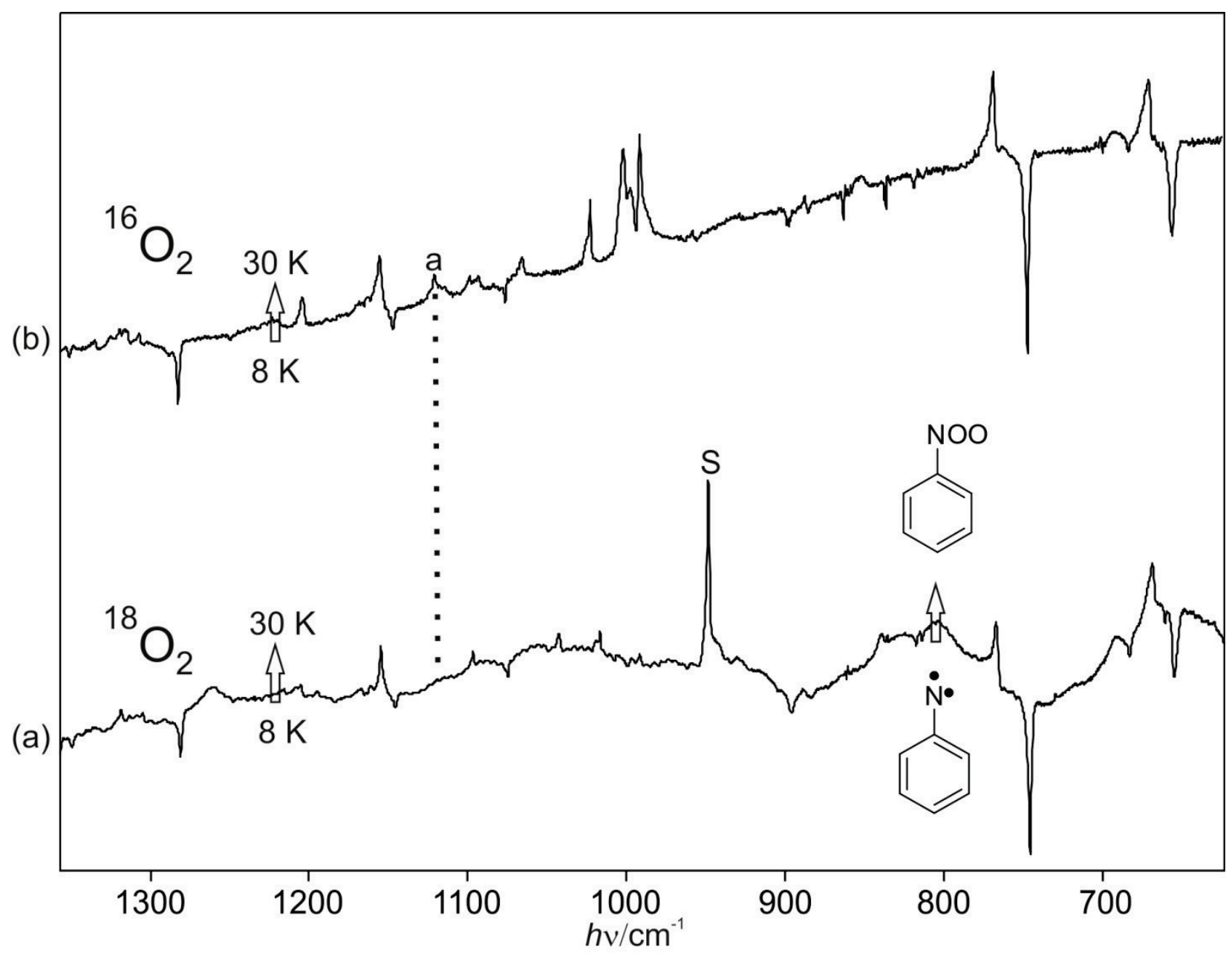

Figure S10. IR spectra showing the reaction of $1 \mathrm{a}$ in $4 \%{ }^{16} \mathrm{O}_{2}$ and ${ }^{18} \mathrm{O}_{2}$-doped $\mathrm{Xe}$ at $30 \mathrm{~K}$ (a) Difference spectrum after annealing at $30 \mathrm{~K}$ of a ${ }^{18} \mathrm{O}_{2}$-doped Xe matrix containing nitrene 1a, bands pointing downward assigned to 1a are decreasing in intensity and bands pointing upward assigned to nitroso oxide $\mathbf{6 a}$ are appearing. The strongest band for the anti-isomer is not appearing in the spectrum (a), it is possibly shifted due to the isotopic labelling $\left(-65 \mathrm{~cm}^{-1}\right.$ according to DFT at the B3LYP/aug-cc-pVTZ level of theory). However, the largest isotopic shift observed was $-52 \mathrm{~cm}^{-1}$, in good agreement with the calculated shift for the $\mathrm{O}-\mathrm{O}$ stretching vibration of the $s y n$-isomer. The rest of the bands were assigned to the $s y n$-isomer based on the calculated shifts. (b) Difference spectrum after annealing at $30 \mathrm{~K}$ of a ${ }^{16} \mathrm{O}_{2}$-doped Xe matrix containing nitrene 1a, bands pointing downward assigned to $\mathbf{1 a}$ are decreasing in intensity and bands pointing upward assigned to the nitroso oxide 6a are appearing. Most intense bands of both isomers are labelled as $\mathbf{a}($ anti) and $\mathbf{s}(s y n)$. 
Table S1. Calculated vibrational frequencies of phenylnitrene 1a at the B3LYP/aug-cc-pVTZ level of theory.

\begin{tabular}{cccc}
\hline \multicolumn{4}{c}{ 1a } \\
\hline mode & sym. & $\tilde{v} / \mathrm{cm}^{-1}$ & $\mathrm{I}_{\mathrm{abs}}{ }^{\mathrm{a}}$ \\
\hline 30 & $\mathrm{~A}_{1}$ & 3201 & 2.5 \\
29 & $\mathrm{~B}_{2}$ & 3199 & 7.1 \\
28 & $\mathrm{~A}_{1}$ & 3191 & 8.6 \\
27 & $\mathrm{~B}_{2}$ & 3175 & 6.6 \\
26 & $\mathrm{~A}_{1}$ & 3169 & 0.3 \\
25 & $\mathrm{~A}_{1}$ & 1586 & 10.5 \\
24 & $\mathrm{~B}_{2}$ & 1557 & 2.3 \\
23 & $\mathrm{~A}_{1}$ & 1464 & 0.8 \\
22 & $\mathrm{~B}_{2}$ & 1446 & 2.1 \\
21 & $\mathrm{~B}_{2}$ & 1342 & 0.1 \\
20 & $\mathrm{~A}_{1}$ & 1311 & 14.8 \\
19 & $\mathrm{~B}_{2}$ & 1269 & 0.7 \\
17 & $\mathrm{~A}_{1}$ & 1170 & 4.8 \\
16 & $\mathrm{~B}_{2}$ & 1093 & 5.8 \\
15 & $\mathrm{~A}_{1}$ & 1027 & 2.5 \\
12 & $\mathrm{~A}_{1}$ & 979 & 0.8 \\
11 & $\mathrm{~B}_{1}$ & 917 & 4.4 \\
10 & $\mathrm{~A}_{1}$ & 833 & 2.2 \\
8 & $\mathrm{~B}_{1}$ & 770 & 50.7 \\
7 & $\mathrm{~B}_{1}$ & 674 & 34.9 \\
5 & $\mathrm{~A}_{1}$ & 525 & 0.3 \\
4 & $\mathrm{~B}_{1}$ & 482 & 2.0 \\
\hline${ }^{\mathrm{a}} \mathrm{Absolute}$ intensities in $\mathrm{km} / \mathrm{mol}$.
\end{tabular}


Table S2. Calculated vibrational frequencies of phenylazide 3 at the B3LYP/aug-cc-pVTZ level of theory.

\begin{tabular}{cccc}
\hline \multicolumn{4}{c}{$\mathbf{3}$} \\
\hline mode & sym. & $\tilde{v} / \mathrm{cm}^{-1}$ & $\mathrm{I}_{\mathrm{abs}}{ }^{\mathrm{a}}$ \\
\hline 36 & $\mathrm{~A}^{\prime}$ & 3199 & 5.1 \\
35 & $\mathrm{~A}^{\prime}$ & 3192 & 12.4 \\
34 & $\mathrm{~A}^{\prime}$ & 3182 & 9.9 \\
33 & $\mathrm{~A}^{\prime}$ & 3174 & 2.3 \\
32 & $\mathrm{~A}^{\prime}$ & 3167 & 2.4 \\
31 & $\mathrm{~A}^{\prime}$ & 2239 & 842.4 \\
30 & $\mathrm{~A}^{\prime}$ & 1637 & 58.5 \\
29 & $\mathrm{~A}^{\prime}$ & 1622 & 6.2 \\
28 & $\mathrm{~A}^{\prime}$ & 1526 & 81.3 \\
27 & $\mathrm{~A}^{\prime}$ & 1489 & 1.1 \\
26 & $\mathrm{~A}^{\prime}$ & 1366 & 104.6 \\
25 & $\mathrm{~A}^{\prime}$ & 1358 & 90.3 \\
24 & $\mathrm{~A}^{\prime}$ & 1326 & 18.2 \\
23 & $\mathrm{~A}^{\prime}$ & 1202 & 2.6 \\
22 & $\mathrm{~A}^{\prime}$ & 1182 & 0.1 \\
21 & $\mathrm{~A}^{\prime}$ & 1156 & 17.4 \\
20 & $\mathrm{~A}^{\prime}$ & 1106 & 8.3 \\
19 & $\mathrm{~A}^{\prime}$ & 1048 & 3.4 \\
18 & $\mathrm{~A}^{\prime}$ & 1019 & 0.6 \\
17 & $\mathrm{~A}^{\prime \prime}$ & 1008 & 0.1 \\
15 & $\mathrm{~A}^{\prime \prime}$ & 923 & 4.9 \\
13 & $\mathrm{~A}^{\prime}$ & 827 & 5.4 \\
12 & $\mathrm{~A}^{\prime \prime}$ & 771 & 59.3 \\
11 & $\mathrm{~A}^{\prime \prime}$ & 700 & 23.4 \\
10 & $\mathrm{~A}^{\prime}$ & 688 & 23.7 \\
9 & $\mathrm{~A}^{\prime}$ & 632 & 0.1 \\
8 & $\mathrm{~A}^{\prime \prime}$ & 561 & 6.9 \\
7 & $\mathrm{~A}^{\prime \prime}$ & 504 & 6.5 \\
6 & $\mathrm{~A}^{\prime}$ & 474 & 0.4 \\
\hline${ }^{\mathrm{a}} \mathrm{Absolute}$ & intensities in $\mathrm{km} / \mathrm{mol}$
\end{tabular}


Table S3. Calculated vibrational frequencies of nitrobenzene 8a at the B3LYP/aug-cc-pVTZ level of theory.

\begin{tabular}{cccc}
\hline \multicolumn{4}{c}{ 8a } \\
\hline mode & sym. & $\tilde{v} / \mathrm{cm}^{-1}$ & $\mathrm{I}_{\mathrm{abs}}{ }^{\mathrm{a}}$ \\
\hline 36 & $\mathrm{~A}_{1}$ & 3225 & 5.0 \\
35 & $\mathrm{~B}_{2}$ & 3225 & 0.5 \\
34 & $\mathrm{~A}_{1}$ & 3196 & 7.7 \\
33 & $\mathrm{~B}_{2}$ & 3188 & 8.7 \\
32 & $\mathrm{~A}_{1}$ & 3175 & 0.7 \\
31 & $\mathrm{~B}_{2}$ & 1650 & 23.4 \\
30 & $\mathrm{~A}_{1}$ & 1628 & 3.1 \\
29 & $\mathrm{~B}_{2}$ & 1575 & 254.3 \\
28 & $\mathrm{~A}_{1}$ & 1514 & 9.9 \\
27 & $\mathrm{~B}_{2}$ & 1491 & 0.7 \\
26 & $\mathrm{~A}_{1}$ & 1369 & 276.4 \\
25 & $\mathrm{~B}_{2}$ & 1351 & 13.5 \\
24 & $\mathrm{~B}_{2}$ & 1342 & 0.3 \\
23 & $\mathrm{~A}_{1}$ & 1196 & 3.6 \\
22 & $\mathrm{~B}_{2}$ & 1184 & 0.7 \\
21 & $\mathrm{~A}_{1}$ & 1117 & 33.3 \\
20 & $\mathrm{~B}_{2}$ & 1100 & 8.1 \\
19 & $\mathrm{~A}_{1}$ & 1044 & 6.9 \\
18 & $\mathrm{~A}_{1}$ & 1024 & 0.2 \\
15 & $\mathrm{~B}_{1}$ & 972 & 3.0 \\
14 & $\mathrm{~A}_{1}$ & 868 & 33.0 \\
12 & $\mathrm{~B}_{1}$ & 822 & 8.8 \\
11 & $\mathrm{~B}_{1}$ & 726 & 78.5 \\
10 & $\mathrm{~A}_{1}$ & 696 & 8.3 \\
9 & $\mathrm{~B}_{1}$ & 688 & 6.2 \\
7 & $\mathrm{~B}_{2}$ & 525 & 1.4 \\
6 & $\mathrm{~B}_{1}$ & 448 & 0.6 \\
4 & $\mathrm{~A}_{1}$ & 395 & 1.0 \\
3 & $\mathrm{~B}_{2}$ & 257 & 0.8 \\
\hline $\mathrm{a} \mathrm{Abs} l u t e$ & $\mathrm{inten}_{1} \mathrm{ities} \mathrm{in} \mathrm{km} / \mathrm{mol}$
\end{tabular}

${ }^{\mathrm{a}}$ Absolute intensities in $\mathrm{km} / \mathrm{mol}$. 
Table S4. Calculated vibrational frequencies of anti-nitroso oxide (anti-6a) and ${ }^{18} \mathrm{O}$-anti-6a at the B3LYP/aug-cc-pVTZ level of theory.

\begin{tabular}{|c|c|c|c|c|c|c|c|}
\hline \multicolumn{4}{|c|}{ anti-6a } & \multicolumn{4}{|c|}{${ }^{18} \mathrm{O}-a n t i-6 \mathbf{a}$} \\
\hline mode & sym. & $\tilde{v} / \mathrm{cm}^{-1}$ & $\mathrm{I}_{\mathrm{abs}}{ }^{\mathrm{a}}$ & mode & sym. & $\tilde{v} / \mathrm{cm}^{-1}$ & $\mathrm{I}_{\mathrm{abs}}{ }^{\mathrm{a}}$ \\
\hline 36 & $\mathrm{~A}^{\prime}$ & 3212 & 1.8 & 36 & $\mathrm{~A}^{\prime}$ & 3212 & 1.8 \\
\hline 35 & $\mathrm{~A}^{\prime}$ & 3204 & 4.4 & 35 & $\mathrm{~A}^{\prime}$ & 3204 & 4.4 \\
\hline 34 & $\mathrm{~A}^{\prime}$ & 3194 & 7.1 & 34 & $\mathrm{~A}^{\prime}$ & 3194 & 7.1 \\
\hline 33 & $\mathrm{~A}^{\prime}$ & 3185 & 6.3 & 33 & $\mathrm{~A}^{\prime}$ & 3185 & 6.3 \\
\hline 32 & $A^{\prime}$ & 3174 & 0.1 & 32 & $\mathrm{~A}^{\prime}$ & 3174 & 0.1 \\
\hline 31 & $\mathrm{~A}^{\prime}$ & 1629 & 16.4 & 31 & $\mathrm{~A}^{\prime}$ & 1628 & 15.9 \\
\hline 30 & $\mathrm{~A}^{\prime}$ & 1612 & 0.8 & 30 & $\mathrm{~A}^{\prime}$ & 1612 & 1.1 \\
\hline 29 & $\mathrm{~A}^{\prime}$ & 1514 & 1.3 & 29 & $\mathrm{~A}^{\prime}$ & 1514 & 1.4 \\
\hline 28 & $\mathrm{~A}^{\prime}$ & 1493 & 9.0 & 28 & $\mathrm{~A}^{\prime}$ & 1493 & 8.7 \\
\hline 27 & $\mathrm{~A}^{\prime}$ & 1361 & 1.6 & 27 & $\mathrm{~A}^{\prime}$ & 1361 & 1.7 \\
\hline 26 & $\mathrm{~A}^{\prime}$ & 1352 & 1.9 & 26 & $\mathrm{~A}^{\prime}$ & 1350 & 3.5 \\
\hline 25 & $\mathrm{~A}^{\prime}$ & 1262 & 12.8 & 25 & $\mathrm{~A}^{\prime}$ & 1261 & 14.6 \\
\hline 24 & $\mathrm{~A}^{\prime}$ & 1209 & 13.2 & 24 & $\mathrm{~A}^{\prime}$ & 1196 & 12.2 \\
\hline 23 & $\mathrm{~A}^{\prime}$ & 1186 & 1.9 & 22 & $\mathrm{~A}^{\prime}$ & 1171 & 3.9 \\
\hline 22 & $\mathrm{~A}^{\prime}$ & 1184 & 1.1 & 23 & $\mathrm{~A}^{\prime}$ & 1184 & 0.6 \\
\hline 21 & $\mathrm{~A}^{\prime}$ & 1135 & 285.8 & 20 & $\mathrm{~A}^{\prime}$ & 1070 & 271.9 \\
\hline 20 & $\mathrm{~A}^{\prime}$ & 1103 & 68.5 & 21 & $\mathrm{~A}^{\prime}$ & 1110 & 20.4 \\
\hline 19 & $\mathrm{~A}^{\prime}$ & 1042 & 0.9 & 19 & $\mathrm{~A}^{\prime}$ & 1042 & 0.3 \\
\hline 17 & $\mathrm{~A}^{\prime}$ & 1016 & 3.5 & 17 & $\mathrm{~A}^{\prime}$ & 1016 & 5.9 \\
\hline 15 & $A^{\prime \prime}$ & 955 & 4.1 & 15 & $A^{\prime \prime}$ & 955 & 4.1 \\
\hline 14 & $\mathrm{~A}^{\prime}$ & 906 & 35.4 & 14 & $A^{\prime}$ & 897 & 45.6 \\
\hline 13 & $A^{\prime \prime}$ & 855 & 0.1 & 13 & $A^{\prime \prime}$ & 855 & 0.1 \\
\hline 12 & $A^{\prime \prime}$ & 789 & 46.1 & 12 & $\mathrm{~A}^{\prime \prime}$ & 789 & 46.3 \\
\hline 11 & $A^{\prime \prime}$ & 690 & 34.4 & 11 & $A^{\prime \prime}$ & 690 & 34.1 \\
\hline 10 & $\mathrm{~A}^{\prime}$ & 678 & 31.3 & 10 & $\mathrm{~A}^{\prime}$ & 670 & 34.4 \\
\hline 9 & $\mathrm{~A}^{\prime}$ & 625 & 1.0 & 9 & $\mathrm{~A}^{\prime}$ & 625 & 1.0 \\
\hline 8 & $\mathrm{~A}^{\prime \prime}$ & 519 & 5.6 & 8 & $A^{\prime \prime}$ & 518 & 5.4 \\
\hline 7 & $\mathrm{~A}^{\prime}$ & 495 & 10.4 & 7 & $\mathrm{~A}^{\prime}$ & 483 & 9.0 \\
\hline 5 & $\mathrm{~A}^{\prime}$ & 404 & 0.4 & 5 & $\mathrm{~A}^{\prime}$ & 393 & 0.5 \\
\hline
\end{tabular}

${ }^{a}$ Absolute intensities in $\mathrm{km} / \mathrm{mol}$. 
Table S5. Calculated vibrational frequencies of syn-nitroso oxide (syn-6a) and ${ }^{18} \mathrm{O}-s y n-6 \mathbf{a}$ at the B3LYP/aug-cc-pVTZ level of theory.

\begin{tabular}{|c|c|c|c|c|c|c|c|}
\hline \multicolumn{4}{|c|}{ syn-6a } & \multicolumn{4}{|c|}{${ }^{18} \mathrm{O}-\operatorname{syn}-\mathbf{6 a}$} \\
\hline mode & sym. & $\tilde{v} / \mathrm{cm}^{-1}$ & $\mathrm{I}_{\mathrm{abs}}{ }^{\mathrm{a}}$ & mode & sym. & $\tilde{v} / \mathrm{cm}^{-1}$ & $\mathrm{I}_{\mathrm{abs}}{ }^{\mathrm{a}}$ \\
\hline 36 & $\mathrm{~A}^{\prime}$ & 3294 & 5.8 & 36 & $\mathrm{~A}^{\prime}$ & 3294 & 5.8 \\
\hline 35 & $A^{\prime}$ & 3205 & 4.0 & 35 & $\mathrm{~A}^{\prime}$ & 3205 & 4.0 \\
\hline 34 & $A^{\prime}$ & 3193 & 8.4 & 34 & $\mathrm{~A}^{\prime}$ & 3193 & 8.4 \\
\hline 33 & $\mathrm{~A}^{\prime}$ & 3185 & 6.7 & 33 & $A^{\prime}$ & 3185 & 6.7 \\
\hline 32 & $A^{\prime}$ & 3172 & 0.2 & 32 & $\mathrm{~A}^{\prime}$ & 3172 & 0.2 \\
\hline 31 & $A^{\prime}$ & 1632 & 26.4 & 31 & $\mathrm{~A}^{\prime}$ & 1631 & 27.1 \\
\hline 30 & $\mathrm{~A}^{\prime}$ & 1591 & 9.4 & 30 & $\mathrm{~A}^{\prime}$ & 1591 & 9.5 \\
\hline 29 & $A^{\prime}$ & 1498 & 0.1 & 29 & $\mathrm{~A}^{\prime}$ & 1498 & 0.1 \\
\hline 28 & $A^{\prime}$ & 1482 & 9.7 & 28 & $A^{\prime}$ & 1482 & 9.8 \\
\hline 27 & $A^{\prime}$ & 1362 & 30.4 & 27 & $A^{\prime}$ & 1361 & 30.3 \\
\hline 26 & $\mathrm{~A}^{\prime}$ & 1345 & 7.3 & 26 & $\mathrm{~A}^{\prime}$ & 1345 & 6.6 \\
\hline 25 & $\mathrm{~A}^{\prime}$ & 1277 & 47.6 & 25 & $\mathrm{~A}^{\prime}$ & 1269 & 45.6 \\
\hline 24 & $\mathrm{~A}^{\prime}$ & 1196 & 16.6 & 24 & $\mathrm{~A}^{\prime}$ & 1192 & 25.5 \\
\hline 23 & $\mathrm{~A}^{\prime}$ & 1186 & 3.7 & 23 & $\mathrm{~A}^{\prime}$ & 1186 & 3.4 \\
\hline 22 & $A^{\prime}$ & 1172 & 35.1 & 22 & $\mathrm{~A}^{\prime}$ & 1158 & 24.9 \\
\hline 21 & $\mathrm{~A}^{\prime}$ & 1112 & 3.3 & 21 & $\mathrm{~A}^{\prime}$ & 1111 & 2.7 \\
\hline 20 & $\mathrm{~A}^{\prime}$ & 1045 & 36.3 & 20 & $\mathrm{~A}^{\prime}$ & 1044 & 7.0 \\
\hline 19 & $\mathrm{~A}^{\prime}$ & 1030 & 201.2 & 16 & $\mathrm{~A}^{\prime}$ & 977 & 205.8 \\
\hline 17 & $\mathrm{~A}^{\prime}$ & 1011 & 13.6 & 17 & $\mathrm{~A}^{\prime}$ & 1008 & 0.0 \\
\hline 15 & $\mathrm{~A}^{\prime \prime}$ & 970 & 3.5 & 15 & $\mathrm{~A}^{\prime \prime}$ & 970 & 3.4 \\
\hline 14 & $\mathrm{~A}^{\prime}$ & 874 & 18.2 & 14 & $\mathrm{~A}^{\prime}$ & 861 & 29.4 \\
\hline 12 & $\mathrm{~A}^{\prime \prime}$ & 798 & 42.5 & 12 & $\mathrm{~A}^{\prime \prime}$ & 797 & 42.5 \\
\hline 11 & $\mathrm{~A}^{\prime}$ & 763 & 0.7 & 11 & $\mathrm{~A}^{\prime}$ & 752 & 0.8 \\
\hline 10 & $\mathrm{~A}^{\prime \prime}$ & 684 & 36.3 & 10 & $\mathrm{~A}^{\prime \prime}$ & 684 & 36.4 \\
\hline 8 & $\mathrm{~A}^{\prime \prime}$ & 586 & 4.0 & 8 & $\mathrm{~A}^{\prime \prime}$ & 580 & 3.9 \\
\hline 7 & $\mathrm{~A}^{\prime}$ & 489 & 9.6 & 7 & $\mathrm{~A}^{\prime}$ & 482 & 8.9 \\
\hline 6 & $\mathrm{~A}^{\prime \prime}$ & 415 & 0.5 & 6 & $\mathrm{~A}^{\prime \prime}$ & 415 & 0.2 \\
\hline 5 & $\mathrm{~A}^{\prime \prime}$ & 412 & 0.8 & 5 & $A^{\prime \prime}$ & 407 & 1.0 \\
\hline 4 & $\mathrm{~A}^{\prime}$ & 344 & 5.4 & 4 & $\mathrm{~A}^{\prime}$ & 334 & 4.9 \\
\hline 3 & $\mathrm{~A}^{\prime}$ & 255 & 1.4 & 3 & $\mathrm{~A}^{\prime}$ & 248 & 1.5 \\
\hline
\end{tabular}

${ }^{\mathrm{a}}$ Absolute intensities in $\mathrm{km} / \mathrm{mol}$. 
Table S6. Calculated harmonic and anharmonic vibrational frequencies of syn-nitroso oxide (syn6a) at the B3LYP/cc-pVTZ level of theory.

\begin{tabular}{cccccccc}
\hline \multicolumn{2}{c}{ syn-6a } & Harmonic & Anharmonic & \multicolumn{2}{c}{ syn-6a } & Harmonic & Anharmonic \\
\hline mode & sym. & $\tilde{v} / \mathrm{cm}^{-1}$ & $\tilde{v} / \mathrm{cm}^{-1}$ & mode & sym. & $\tilde{v} / \mathrm{cm}^{-1}$ & $\tilde{v} / \mathrm{cm}^{-1}$ \\
\hline 36 & $\mathrm{~A}^{\prime}$ & 3297.0 & 3160.8 & 18 & $\mathrm{~A}^{\prime \prime}$ & 1028.3 & 1005.2 \\
35 & $\mathrm{~A}^{\prime}$ & 3205.7 & 3074.8 & 17 & $\mathrm{~A}^{\prime}$ & 1012.1 & 994.0 \\
34 & $\mathrm{~A}^{\prime}$ & 3193.5 & 3044.6 & 16 & $\mathrm{~A}^{\prime \prime}$ & 1007.7 & 987.1 \\
33 & $\mathrm{~A}^{\prime}$ & 3185.0 & 3072.5 & 15 & $\mathrm{~A}^{\prime \prime}$ & 965.8 & 946.9 \\
32 & $\mathrm{~A}^{\prime}$ & 3172.6 & 3021.3 & 14 & $\mathrm{~A}^{\prime}$ & 876.3 & 863.7 \\
31 & $\mathrm{~A}^{\prime}$ & 1634.4 & 1594.9 & 13 & $\mathrm{~A}^{\prime \prime}$ & 858.4 & 841.4 \\
30 & $\mathrm{~A}^{\prime}$ & 1594.6 & 1552.6 & 12 & $\mathrm{~A}^{\prime \prime}$ & 799.7 & 783.0 \\
29 & $\mathrm{~A}^{\prime}$ & 1500.8 & 1464.6 & 11 & $\mathrm{~A}^{\prime}$ & 764.4 & 751.7 \\
28 & $\mathrm{~A}^{\prime}$ & 1484.2 & 1454.1 & 10 & $\mathrm{~A}^{\prime \prime}$ & 692.2 & 678.3 \\
27 & $\mathrm{~A}^{\prime}$ & 1362.2 & 1325.2 & 9 & $\mathrm{~A}^{\prime}$ & 626.7 & 618.0 \\
26 & $\mathrm{~A}^{\prime}$ & 1345.5 & 1314.8 & 8 & $\mathrm{~A}^{\prime \prime}$ & 587.4 & 574.0 \\
25 & $\mathrm{~A}^{\prime}$ & 1276.3 & 1230.4 & 7 & $\mathrm{~A}^{\prime}$ & 489.6 & 481.2 \\
24 & $\mathrm{~A}^{\prime}$ & 1197.4 & 1173.4 & 6 & $\mathrm{~A}^{\prime \prime}$ & 417.0 & 408.6 \\
23 & $\mathrm{~A}^{\prime}$ & 1188.9 & 1171.5 & 5 & $\mathrm{~A}^{\prime \prime}$ & 413.5 & 396.6 \\
22 & $\mathrm{~A}^{\prime}$ & 1173.3 & 1143.6 & 4 & $\mathrm{~A}^{\prime}$ & 344.0 & 338.8 \\
21 & $\mathrm{~A}^{\prime}$ & 1113.7 & 1103.3 & 3 & $\mathrm{~A}^{\prime}$ & 255.3 & 249.2 \\
20 & $\mathrm{~A}^{\prime}$ & 1049.9 & 1030.1 & 2 & $\mathrm{~A}^{\prime \prime}$ & 160.1 & 150.6 \\
19 & $\mathrm{~A}^{\prime}$ & 1040.7 & 1016.8 & 1 & $\mathrm{~A}^{\prime \prime}$ & 75.9 & 64.6 \\
\hline
\end{tabular}


Table S7. Combination bands between $900-1100 \mathrm{~cm}^{-1}$ for syn-nitroso oxide (syn-6a) calculated at the B3LYP/cc-pVTZ level of theory (anharmonic).

\begin{tabular}{cccccccc}
\hline \multicolumn{2}{c}{ Fundamental } \\
mode & mode & $\begin{array}{c}\text { Combination band } \\
\tilde{v} / \mathrm{cm}^{-1}\end{array}$ & $\mathrm{I}_{\mathrm{abs}}{ }^{\mathrm{a}}{ }^{\mathrm{a}}$ & \multicolumn{2}{c}{ Fundamental } \\
mode & mode & $\begin{array}{c}\text { Combination band } \\
\tilde{v} / \mathrm{cm}^{-1}\end{array}$ & $\mathrm{I}_{\mathrm{abs}}{ }^{\mathrm{a}}$ \\
\hline 2 & 11 & 902.4 & 0.00 & 6 & 9 & 1025.8 & 0.04 \\
1 & 13 & 906.1 & 0.02 & 3 & 12 & 1032.0 & 0.00 \\
4 & 8 & 912.7 & 0.00 & 1 & 16 & 1049.9 & 0.02 \\
1 & 14 & 927.0 & 0.05 & 7 & 8 & 1054.5 & 0.00 \\
3 & 10 & 927.2 & 0.00 & 1 & 17 & 1057.2 & 0.00 \\
2 & 12 & 932.8 & 0.50 & 1 & 18 & 1068.2 & 0.02 \\
4 & 9 & 956.8 & 0.02 & 6 & 10 & 1076.4 & 0.15 \\
5 & 8 & 970.7 & 3.18 & 5 & 10 & 1078.9 & 0.07 \\
6 & 8 & 980.6 & 0.21 & 1 & 19 & 1080.5 & 0.11 \\
2 & 13 & 991.5 & 0.08 & 4 & 11 & 1088.5 & 0.93 \\
3 & 11 & 1000.0 & 2.43 & 3 & 13 & 1090.4 & 0.00 \\
1 & 15 & 1010.7 & 0.01 & 1 & 20 & 1093.8 & 0.08 \\
2 & 14 & 1013.2 & 0.00 & 2 & 15 & 1096.0 & 0.38 \\
4 & 10 & 1017.0 & 0.00 & 7 & 9 & 1100.0 & 1.26 \\
5 & 9 & 1018.1 & 0.00 & & & & \\
\hline
\end{tabular}

${ }^{\mathrm{a}}$ Absolute intensities in $\mathrm{km} / \mathrm{mol}$ 